\title{
Effects of Access to a Naturally Reinforcing Activity on the Acquisition of Repertoires in Autistic Learner
}

\author{
Daniel Carvalho de Matos $^{1 *_{2}} \quad$ Patrícia Maria Sombreira Araújo ${ }^{1}$ \\ Flor de Maria Araújo Mendonça Silva ${ }^{1}$ Wellyson da Cunha Araújo Firmo ${ }^{12}$ \\ Pollianna Galvão Soares de Matos ${ }^{12}$ \\ 1.CEUMA University, Brazil \\ 2.Federal University of Maranhão, Brazil
}

\begin{abstract}
Applied Behavior Analysis (ABA) to Autism Spectrum Disorder is concerned in arranging reinforcing contingencies, which favor the learning of important repertoires. In the literature, the use of preferred arbitrary reinforcers is emphasized to shape and strengthen impaired targets. This may increase the likelihood that learners will cooperate with demands. Once a child is more exposed to learning contingencies, and becomes more fluent in certain repertoires, access to arbitrary reinforcers should be made more intermittently. It is possible, this way, that engaging in a given activity be now maintained by a natural or automatic reinforcing effect, depending solely on the learner. This research had the purpose of assessing if, by allowing access to a task, which previously became motivating for a child with ASD (pairing colored blocks), other non-verbal skills could be shaped (visually pairing similar pictures; motor imitation with toys; pairing pictures to their corresponding dictated names). The study was conducted in a laboratory from a private University in Brazil. A multiple probe design, across different target repertoires, was used to ensure experimental control by the task of pairing similar colored blocks. As a result, it was demonstrated that all target repertoires were learned, suggesting that access to the previously mastered task, possibly maintained by natural reinforcement, solely established three different repertoires. When the child's parents were interviewed to give their perception on the use of pairing blocks as reinforcer, they were more satisfied than in the case that other reinforcers, such as videos and edibles, are used.
\end{abstract}

Keywords: autism spectrum disorder, arbitrary reinforce, natural reinforcer

DOI: $10.7176 /$ RHSS/10-22-06

Publication date: November $30^{\text {th }} 2020$

\section{Introduction}

Children with an Autism Spectrum Disorder (ASD) diagnosis commonly show impairment in non-verbal and verbal repertoires, which are important for development. Principles of Applied Behavior Analysis (ABA) are commonly used to teach the repertoires, so the children may become more functional and independent in social contexts. The addressed skills are also called operants in Behavior Analysis. An operant is a kind of behavior shaped and maintained by consequences, and it can be non-verbal or verbal (Skinner, 1992). As an example of non-verbal operant with which interventionists are usually concerned, there is motor imitation. When an interventionist is teaching such skill, he/she may perform the action of clapping hands as a model for a given child. After that, the learner should emit the response of clapping hands, according to the presented model. In the end, the teacher praises the child and gives him/her an opportunity to access a preferred item, which may be an edible, or some kind of fun activity, depending on the child's interests (Greer \& Ross, 2008; Lovaas, 2003; Matos, 2016; Sundberg \& Partington, 1998).

It is important to mention that, whenever a child makes a mistake during a trial, the teacher should provide a correction. In the case of the mentioned example, he/she should manipulate physical prompts, which may be faded out along more opportunities to respond, so the learner may become more independent. Similar procedures are used to teach language, that is, verbal repertoires. An important case is related to the skill of making verbal requests. If a given child shows interest in a fun video, for example, the interventionist may provide opportunities for the child to ask for the video, so he/she has access to it. Suppose the child shows very limited skills regarding communication with spoken words and sentences. Nevertheless, consider that, in the case of this given learner, an important prerequisite exists: vocal imitation. When a teacher provides the child with an opportunity to ask for a desired cookie, for example, he/she may say "cookie, please", and the teacher, then, delivers the cookie. Whenever necessary, the teacher delivers the vocal model "cookie, please", so the child may repeat it. Along several opportunities to respond, it is hoped that the child will become more independent in making requests without prompts (Greer \& Ross, 2008; Lovaas, 2003; Matos, 2016; Sundberg \& Partington, 1998).

The examples of repertoires (non-verbal and verbal) mentioned frequently are among the skills an interventionist aims to develop in many learners with ASD. Since, in the case of many learners, several repertoires are not consistently demonstrated, specialized procedures should be planned and implemented to address the issue. ABA interventions may be conducted in the form of massed trials (discrete trial teaching - DTT), so the learners may have many opportunities to respond and be reinforced. Along the opportunities, prompts may be manipulated 
and faded out gradually in order to establish independent performance (Matos, 2016; Matos \& Matos, 2018; Matos, Hora, Rubim \& Matos, 2020; Matos, Matos, Hora, 2020).

During the establishment of repertoires through DTT, it is common practice to teach through the use of arbitrary reinforcers, which are not necessarily functionally related to the responses they aim to establish. As an example, consider the case of a child to whom it is provided the dictated word "car", so he/she may repeat it (vocal imitation). When the child does so, the teacher delivers a piece of candy as a reinforcing consequence, instead of handing over a toy car. It might the case that this child is much more motivated by the piece of candy, and this is taken into consideration by the teacher, once powerful motivating operations need to be established in many cases of children with ASD, who are reluctant in attending demands (Allen \& Cowan, 2008; Tarbox \& Najdowski, 2008).

Arbitrary reinforcers are frequently used during the teaching of repertoires through DTT. Surely, this approach proved to be effective in teaching skills to learners with ASD and other learning disabilities, increasing also their potential to acquire new skills in more natural contexts and without discrete trial procedures. However, DTT has been criticized in the past due to the fact that arbitrary reinforcers, unrelated to the responses they aim to establish, are frequently used instead of more natural reinforcers functionally related to the target responses. It appears that some interventionists consider that the use of more natural reinforcers in natural environments should be preferred over arbitrary ones (Allen \& Cowan, 2008). Nevertheless, the literature also states that both approaches should be used in combination to establish functional skills in learners with atypical development (Sundberg \& Partington, 1998).

According to Moreira and Medeiros (2007), a reinforcer is called natural when it is the direct product of someone's behavior. Conversely, a reinforcer is called arbitrary when it is an indirect product of someone's behavior. To illustrate these cases, the mentioned authors considered the behavior of a musician playing a guitar. When the person is playing alone in a bedroom, the behavior is likely to be maintained by the music itself, as a kind of natural reinforcer. However, once the musician plays his guitar in a bar for money, the behavior is probably maintained by the payment as an arbitrary reinforcer.

In the literature, a natural reinforcer is also called automatic. It does not rely on social mediation. A behavior maintained by a natural/automatic reinforcer may be either non-verbal or verbal (Barros \& Benvenutti, 2011; Vaughan \& Michael, 1982). There are behaviors, emitted by children with ASD, which are usually maintained by automatic reinforcers. That may be the case of some repetitive/stereotypic behavior patterns that many children show. Functional analysis approaches may help determining the kind of reinforcing consequence that controls the behavior (Neef \& Petterson, 2007).

In the literature, some authors also suggest that automatic reinforcers are responsible for the establishment of language/verbal behavior in the beginning of children's lives. According to Smith, Michael and Sundberg (1996), for example, when a mother is interacting with her little child in a ludic and warm manner, she is frequently providing stimuli, such as verbalizations to develop vocal behavior. During the interactions, a given mother constantly presents the verbal demand "say mommy", while she smiles and touches the child in a gentle manner. In other words, the stimuli (the verbal model, smile and physical contact) are being paired. It is considered that social stimuli, such as physical contact and smiles provided by mothers, are powerful reinforcers for their children. Smith et al. suggested that the pairing process could be responsible in establishing a reinforcing function to the spoken word "mommy". With time, it could be possible to hear a child saying "mommy" more frequently. It is supposed that, in many occasions in which the child emits the verbalization, he/she does so because of the newly acquired reinforcing properties of the spoken word "mommy". In other words, the audible properties of the spoken word could be now an automatic reinforcer, responsible for maintaining the child's vocal behavior.

It was previously stated that arbitrary reinforcers are important in ABA interventions developed for learners with ASD, considering especially the case of the DTT format (Matos, 2016; Matos \& Matos, 2018; Matos, Hora, et al., 2020; Matos, Matos, et al., 2020). Many learners require the use of powerful arbitrary reinforcers to increase the likelihood that they will cooperate with demands. Initially, the reinforcers should be contingent to every independent response along many trials. Along the exposure, the learners should become more skilled and commit few or no errors. When this happens, the schedule of reinforcement should be changed in a manner that arbitrary reinforcers are now provided intermittently. This is important, considering that learners should not become dependent on receiving a reinforcer for every appropriate response they emit. The life outside an analog context, such as a laboratory or clinic, does not function that way. ABA interventions with a DTT format are important to shape non-verbal and verbal repertoires, and they help developing consistency in responding. Generalization measures of the acquired skills to more natural environments should also be obtained. It is hoped that eventually repertoires, previously acquired through DTT and use of arbitrary reinforcers, be emitted solely under the control of natural learning effects.

Some parents of children with ASD mention discomfort with the use of some arbitrary reinforcers to develop skills. Common preference assessments in ABA (DeLeon \& Iwata, 1996) frequently show that tangible items, such as tablets and cell phones, and edibles are powerful reinforcers for many children with ASD. The scientific literature has already demonstrated the efficacy of arbitrary reinforcers in establishing basic and more advanced 
skills. Once a given repertoire is established, and the use of arbitrary reinforcers is progressively faded out, it is possible that the repertoire itself became naturally reinforcing for a learner. In this sense, it could be argued that allowing access to the previously established repertoire could be used as a reinforcer to shape and strengthen new skills in a given learner. It is supposed that this approach could help diminishing parents' discomfort regarding the use of arbitrary reinforcers, such as tablets and edibles to shape skills in their children. It is important to argue, though, that making the access to a previously acquired task contingent to compliance of new demands is also an arbitrary relation.

The purpose of this study was to assess whether, making the access to a previously established and preferred task (pairing colored blocks) contingent to the compliance of new tasks, successfully established other non-verbal repertoires in a 8 year old boy with ASD. In parallel, his parents were interviewed as to their perception on the use of a task, such as pairing colored blocks, as a reinforcer to teach new skills to the child.

\section{Method}

This study consisted of an experimental investigation, conducted in a research laboratory from a private University in Brazil. An experimental research comprises the manipulation of independent variable (IV), and assessment of the effects on a dependent variable (DV). In this study, the primary DV consisted of the following: 1) percentage of correct responses of pairing similar pictures; 2) percentage of correct responses of motor imitation with toys; 3 ) percentage of correct responses of pairing pictures to their corresponding dictated names. The IV consisted of allowing a task of pairing colored blocks as a possible reinforcer. During a training condition, opportunities to pair colored blocks were provided contingent to correct responses, regarding the mentioned DV. Along several trials, whenever an incorrect response was emitted (or no response occurred during an allotted time), correction procedures described later were implemented.

To ensure experimental control of the IV over the DV, a variation of multiple baseline design, multiple probe design (Cooper, Heron \& Heward, 2006; Pereira, Shitsuka, Parreira \& Shitsuka, 2018), was used. Experimental control was obtained across three different repertoires (the previously mentioned DV) for a single participant. At first, a probe was conducted for each repertoire. After that, a baseline condition was applied to one of the repertoires and, once it was determined that the participant emitted few or no correct responses, an intervention condition with reinforcement of correct performance and error correction when needed, was implemented. After an arbitrary learning criterion was achieved, new probes were conducted for all repertoires. Then, the same sequence of conditions, regarding baseline and intervention, were replicated with the second repertoire. After the achievement of an arbitrary learning criterion, all repertoires were probed again. Finally, the same sequence of conditions, baseline and intervention, was replicated with the third repertoire as well. When the third repertoire was acquired, new probes for all repertoires, with a new set of stimuli for each of them to measure generalization, were administered.

\subsection{Participant}

The research participant was an 8-year-old boy with ASD. He was attended through ABA interventions twice a week in a research laboratory from a private University in Brazil (CEUMA University). By the time data collection was taken, he was able to pair blocks according to their colors without errors. This was a kind of activity to which no extrinsic reinforcement was necessary to maintain the participant engaged. He was also able to pair several pictures to their corresponding models. Among the skills the child did not demonstrate accurately, and that were targeted in DTT programs, there were the following: pairing pictures to their similar corresponding models; demonstrating motor responses based on models provided by a therapist; pairing pictures to their corresponding dictated models. The child was not able to establish communication with words. Curricular goals, based on the criteria of assessment protocols, were established to teach non-verbal and verbal Skills (Sundberg, 2008).

\subsection{Environment}

During the course of the study, experimental sessions were held in the context of the Assessment, Research and Intervention in Autism Spectrum Disorder Laboratory (LAPITEA), situated in a private Brazilian University (CEUMA University). The room, where data collection happened, was equipped with a table and chairs. An experimenter and child remained seated on the chairs, facing each other.

\subsection{Instruments and materials}

For the tasks of visually pairing similar pictures and pairing pictures to their corresponding dictated names, plasticized cards, measuring $6 \times 3 \mathrm{~cm}$ and containing images related to different categories (e.g., animals, transportation), were used. For the task of motor imitation with toys, stimuli such as little cars, balls and animals were used. Each Correct response, in these tasks during a training condition, resulted in an opportunity to pair a block from an array to its corresponding model with the same color. Datasheets were organized to take data on the performance of each participant during assessments and interventions. 
For each of the repertoires corresponding to DV of the research, two different groups of targets were established (G1 and G2). G1 comprised targets to be assessed and trained. Targets from G2, on the other hand, were solely probed for generalization reasons. As to the targets, considering the repertoire of visually pairing similar pictures, G1 involved pairing a dog breed to another; pairing a car model to a new model; and pairing a bike model to a new model. G2 consisted of new exemplars of the same categories already mentioned.

Regarding the targets of pairing pictures to their dictated models, the stimuli in the G1 group also consisted of a dog breed, a car model and bike model. Stimuli in the G2 group were variations of those from G1. Finally, in the case of motor imitation with toys, the stimuli in the G1 group included a little car, a ball and a horse. The stimuli in the G2 group were variations of those from G1.

\subsection{Procedure}

The study comprised three phases (assessment probe, baseline and treatment). Each of them is presented separately as follows:

First phase. Assessment probe. During first phase, an assessment probe was conducted with all repertoires defined as DV (visually pairing similar pictures; motor imitation; pairing pictures to their dictated names), including groups G1 and G2. For each task, the probe consisted of a session with 12 discrete trials. During a trial of visually pairing similar pictures, the experimenter placed an array of three pictures on a table, and close to the child, who was sitting next to the pictures. Then, another picture, similar to one from the array, was shown as a model. The child had up to $3 \mathrm{~s}$ to point to the corresponding picture from the array.

During a trial of the motor imitation task, the experimenter had to demonstrate a motor response (e.g., clapping hands) as a model for the child, and presented the verbal instruction "do the same". The child had up to $3 \mathrm{~s}$ to emit the same response as the model. Finally, during a trial of pairing pictures to their corresponding dictated names, the experimenter placed an array with three different pictures on the table close to the child. The name of a picture was presented out loud as a model, so the child could point to the corresponding picture from the array. Just as in the case of the other tasks, a response had to be emitted within $3 \mathrm{~s}$. During this phase of the study, no differential consequences were applied, that is, correct responses were not reinforced and incorrect responses were not prompted.

It is important to mention that, after treatment phase (third phase described later) started, new probes were also conducted for all repertoires. For each of them, all probes involved targets from both groups G1 and G2. G1 comprised the targets, which were addressed in treatment phase. G2 comprised targets, which were never taught. They were variations of those from G1. Considering that they were also probed after treatment started, these probes served as measures of generalization.

Second phase. Baseline. This phase was similar to the previous one. However, several sessions of 12 discrete trials were administered for each repertoire defined as DV. This was done to ensure that the child would not show a spontaneous improvement on the repertoires, before the implementation of a treatment condition. In this phase, the responses did not produce differential consequences for correct and incorrect responses as well.

Third phase. Treatment. In this phase, each of the repertoires defined as DV was taught. The trials were conducted in the same manner that was applied to first and second phases. Nevertheless, differential consequences were indeed applied to correct and incorrect responses. Regarding correct responses for all tasks, the consequence for the child consisted solely in an opportunity to pair blocks according to the color, as a kind of reinforcer. In the case of incorrect responses (or no response within 3s), specific procedures were conducted for each task.

To address incorrect performance regarding a trial to visually pair similar pictures, the experimenter pointed to the picture from an array, which corresponded to a presented model. If that was not enough for the child to respond, the experimenter gently put the child's hand on the right picture. Similar corrections were established for the case of pairing pictures to their corresponding dictated names. In the case of a motor imitation trial, the experimenter provided a partial physical prompt. If the trial was related to the response of clapping hands for instance, the experimenter gently positioned each of the child's hands close to each other, so the child could complete the action of clapping hands. However, if that were not enough, a full physical prompt would be provided. In the mentioned example, the experimenter would fully guide the child, physically and gently, to perform the action of clapping hands. An arbitrary learning criterion was defined in order to end the treatment condition: either the child had to emit at least $80 \%$ correct responses, regarding each of the three tasks in two consecutive sessions of 12 trials, or he had to emit $100 \%$ correct responses in a single session.

\subsection{Ethical procedures}

This study was approved by an ethics committee, in research with humans (authorization No. 2.145.293), from CEUMA University, Brazil, São Luís-MA.

\section{Results}

Figure 1 shows the percentage of correct responses along three phases (probe, baseline and treatment), and 
regarding three different repertoires defined as DV for the child, who participated in this study.



Figure 1. Percentage of correct responses of pairing similar pictures (task 1 - upper graph), motor imitation (task 2 - middle graph) and pairing pictures to their corresponding dictated names (task 3 - lower graph) by the participant. Data was taken across the following phases: probe; baseline; treatment.

As it can be seen in Figure 1 regarding tasks 1, 2 and 3, the research participant emitted 4\%, 8\% and $0 \%$ correct responses, respectively, during a first probe (related to G1 and G2 targets). When baseline commenced for task 1 , he emitted $8 \%$ correct responses after four sessions. A treatment phase started for this task, but nothing was done to tasks 2 and 3 until treatment produced acquisition of the repertoire related to task 1 . An arbitrary learning criterion was achieved after six sessions (no errors occurred).

Thereafter, a new probe was conducted for all tasks. For task 1, the probe showed no errors, considering both G1 and G2 targets. In other words, the emergence of untaught targets (G2) was demonstrated. As to the new probe for tasks 2 and 3, no correct responses were emitted. A baseline phase started for task 2 and, after three sessions, no correct responses were emitted. Meanwhile, no procedure was held for task 3 . The treatment phase was carried out for task 2 after baseline. In seven sessions, 100\% correct responses were emitted and the phase was terminated. Later, another probe was again conducted for all tasks. Once again, no errors were committed in task 1 . In the case of task 2, 92\% correct responses occurred, indicating the emergence of untaught targets as well. No correct performance was demonstrated in the probe, considering the case of task 3.

When baseline and treatment phases were established for task 3, intervention for tasks 1 and 2 was withheld. As to the case of task 3, no correct responses were demonstrated after three baseline sessions. When the treatment phase started, nine sessions were necessary to achieve the learning criterion. Finally, a final probe was performed for all tasks. As to tasks 1 and 2, this also served to measure maintenance of the repertoires, several sessions after intervention was discontinued. No errors were demonstrated in task 1, replicating data from previous probes after treatment. Performance, regarding task 2 , was also the same as in the previous probe, that is, $92 \%$ correct responses. In the case of task 3, 67\% correct responses were emitted. All errors were related to untaught targets. In other words, the emergence effect was lower compared to the other tasks.

\section{Discussion}

Data from the current research strongly indicated that, allowing access to a preferred task of pairing colored blocks, successfully established three different repertoires, defined as DV, in a 8 year-old child with ASD. During the treatment phase, no other variable was used as a reinforcer to teach the target repertoires. The multiple probe 
design used also indicated the establishment of experimental control, considering that each of the target repertoires were acquired solely when treatment was defined (Cooper et al., 2006).

In the past, pairing colored blocks used to be a skill that the participant did not have. Arbitrary reinforcers were initially used to establish this skill but, with time, they were no longer needed. In that case, it is supposed that the repertoire was now maintained by natural reinforcement. The results of this study suggest that, when doing a given activity is no longer dependent on any extrinsic or arbitrary reinforcement, opportunities to engage in that activity may be used as a reinforcer to shape new skills. That is exactly what was done. It is true, however, that other types of preferred reinforcers (e.g., videos and edibles) could be used instead to produce the same effect, or better, of establishing other skills through DTT (Matos, 2016; Matos \& Matos, 2018; Matos, Hora, et al., 2020; Matos, Matos, et al., 2020).

The main reason for the conduction of the study was the parent's complaints as to the use of arbitrary and tangible reinforcers to establish repertoires in the child. Many parents, actually, usually report discomfort about using reinforcers, such as videos and edibles, to make their children with ASD become better respondents in several tasks. They expect their children to be able to respond solely as a function of social stimuli, such as verbal praise and gentle physical touch. That was exactly what the parents of the research participant (the child with ASD) expected to happen. When data collection with the research participant ended, his parents were interviewed, as to give their perception on the use of a previously mastered task, from an individualized curriculum, as a reinforcer to develop other skills. They said they were relieved to know about that, and acknowledged the efficacy and efficiency of ABA interventions in teaching skills that improved the child's development and quality of life. This information was viewed as measure of social validity of the interventions.

According to Baer, Wolf and Risley (1968), interventions in ABA must attend some basic criteria. Among them, it is known that the interventions need to be applied, seeking the improvement of socially relevant behaviors. The interventions also need to be effective, in the sense that behavior change shall be remarkable and socially valid. In the case of learners with ASD, assessments on the perceptions, of those responsible for them, are essential. In a recent study, for example, Sousa, Silva, Ramos and Freitas (2020) assessed parent's perception on ABA interventions conducted to their children with ASD. According to the authors, parent's perception confirms the ABA as a science that seek to identify, assess and change behaviors and skills, which need to be improved or reduced. The parents acknowledged that ABA is effective and concerned with the improvement of socially accepted behaviors and reducing inappropriate behaviors, such as repetitive behaviors and stereotypes.

\section{Final Considerations}

In this study, it was demonstrated that, by allowing opportunities to engage in an activity maintained by natural reinforcement, three different repertoires were successfully established in a child with ASD. During ABA interventions, specialized assessments are frequently conducted to select stimuli, which may be used as reinforcers to shape new repertoires in learners with ASD. It is common that, among the most preferred stimuli, there are devices such as cell phones, tablets and edibles. When they are used as reinforcers, they may be very important in establishing non-verbal and verbal repertoires, which are commonly impaired in many learners. As it was said before, in interventions related to DTT, the teaching is organized in massed trials per target repertoire. Access to a reinforcer is provided contingent to each correct response, initially. With time, the reinforcement becomes more intermittent and it is hoped that, at some point, the responses may be emitted under the control of natural learning effects.

Although this process might facilitate a given learner's development significantly, some parents show discomfort about using certain arbitrary reinforcers (e.g., edibles and videos) to teach skills to their children with ASD. This study may be considered scientifically and socially relevant, because it was demonstrated that the use of a previously mastered skill from the participant's curriculum functioned as a reinforcer, which shaped three new repertoires. The parents reported that they were more satisfied with the use of the new type of reinforcer, which served as a measure of social validity of the intervention. It was concluded that the type of reinforcer of the current study could be an alternative to the use of certain arbitrary reinforcers, which many parents dislike. New investigations on this theme should be conducted with more learners with ASD, as to test the generality of the intervention.

\section{References}

Allen, K. D., \& Cowan, R. J. (2008). Naturalistic Teaching Procedures. In J.K. Luiselll, D.C. Russo, W.P. Christian \& S.M. Wilczynski (Eds.). Effective Practices for Children with Autism. (pp.240-270). New York: OXFORD.

Baer, D. M., Wolf, M.M., \& Risley, T.R. (1968). Some current dimensions of applied behavior analyisis. Journal of Applied Behavior Analysis, 1, 91-97.

Barros, T., \& Benvenutti, M.F.L. (2011). Reforçamento automatic: estratégias de análise e intervenção. Acta Comportamentalia, 20, 177-184.

Cooper, JO, Heron, TE \& Heward, WL. (2006). Applied behavior analysis. Second edition. New Jersey: Pearson 
Merry Prentice Hall.

DeLeon, I.G., \& Iwata, B.A. (1996). Evaluation of a multiple-stimulus presentation format for assessing reinforcer preferences. Journal of Applied Behavior Analysis, 29, 519-533.

Greer, RD \& Ross, DE. (2008). Verbal Behavior Analysis: Inducing and expanding complex communication in children with severe language delays. Boston: Allyn \& Bacon.

Lovaas, OI. (2003). Teaching individuals with developmental delays. Austin, TX: Pro-Ed.

Matos, DC. (2016). Análise do comportamento aplicada ao desenvolvimento atípico com ênfase em autismo. Porto Velho: AICSA.

Matos, D.C., Hora, A.F.T., Rubim, A.L., \& Matos, P.G.S. (2020). Case study regarding behavioral interventions to a child with autism spectrum disorder. International Journal of Development Research, 10, 35825-35828.

Matos, D.C., \& Matos, P.G.S. (2018) Intervenções em psicologia para inclusão escolar de crianças autistas: estudo de caso. Revista Espaço Acadêmico, 211,21-31.

Matos, D.C., Matos, P.G.S., Hora, A.F.T. (2020). Avaliação, intervenção e assessorial em psicologia educacional ao transtorno do espectro autista: experiência do LAPITEA. In: A.T. Bolsoni-Silva, D. Zilio, H.L. Gusso, J.H. Almeida, P.C.M. Mayer (Orgs.), Comportamento em foco (Vol. 10, pp. 140-165). São Paulo: Associação Brasileira de Psicologia e Medicina Comportamental, ABPMC.

Moreira, M. B., \& Medeiros, C. A. (2007). Princípios Básicos de Análise do Comportamento. Porto Alegre: Artmed.

Neef, A.N., \& Peterson, S.M. (2007). Functional Behavior Assessment. In J.O. Cooper, T.E. Heron \& W.L. Heward (Eds.), Applied Behavior Analysis. (pp. 500 - 523). New Jersey: Pearson Education.

Pereira, A.S., Shitsuka, D.M., Parreira, F.J., \& Shitsuka, R. (2018). Metodologia do trabalho cientifico. Santa Maria: UAB / NTE / UFSM.

Skinner, B. F. (1992). Verbal behavior. Englewood Cliffs, NJ: Prentice Hall. (Obra original publicada em 1957).

Smith, R., Michael, J., \& Sundberg, M.L. (1996). Automatic reinforcement and automatic punishment in infant vocal behavior. The Analysis of Verbal Behavior, 13, 39 - 48.

Sousa, D.L.D., Silva, A.L., Ramos, C.M.O., \& Melo, C.F. (2020). Análise do comportamento aplicada: a percepção de pais e profissionais acerca do tratamento em crianças com espectro autista. Contextos Clínicos, $13,105-124$.

Sundberg, M.L. (2008). The verbal behavior milestones assessment and placement program: The VB-MAPP (2nd ed.). Concord, CA: AVB Press.

Sundberg, M.L., \& Partington, J.W. (1998). Teaching language to children with autism or other developmental disabilities. Danville, CA: Behavior Analysts, Inc.

Tarbox, R.S.F., \& Najdowski, A.C. (2008). Discrete trial training as a teaching paradigm. In J.K. Luiselll, D.C. Russo, W.P. Christian \& S.M. Wilczynski (Eds.). Effective Practices for Children with Autism. (pp.204-219). New York: OXFORD.

Vaughan, M. E., \& Michael, J. L. (1982). Automatic Reinforcement: an important but ignored concept. Behaviorism, 10, 217-227. 